\title{
Upper Limb Arterial Ischemia Occurred After Accidental Antibiotics Intra-Arterial Injection: Report of Three Cases
}

Elfatemi B*, Ouldaselek E, Tijani Y, Lazraq M, Khloufi S, Idrissi R, Sefiani Y, Lekehel B, Mesnaoui A, Ammar F, Bensaid Y

Service de chirurgie vasculaire hôpital Ibn Sina Rabat

DOI: $10.36347 /$ simcr.2020.v08i03.010

| Received: 29.02.2020 | Accepted: 07.03.2020 | Published: 10.03.2020

*Corresponding author: Elfatemi Bouthainah

Abstract

The administration of antibiotics by injection may have some consequences as loco regional reactions ranging from skin necrosis at the injection site to acute limb ischemia. The observations of three patients with acute upper limb ischemia following accidental intra-arterial antibiotic injection with coldness, cyanosis, suppression of radial and ulnar pulses and necrosis of the extremities are presented there. Enoxaparin-based heparin therapy was initiated with very good results. Through these observations, and after a review of the literature, the pathophysiological mechanisms and therapeutic means will be reminded.

Keywords: Antibiotics -ischemia-necrosis-heparin therapy-intra arterial.

Copyright @ 2020: This is an open-access article distributed under the terms of the Creative Commons Attribution license which permits unrestricted use, distribution, and reproduction in any medium for non-commercial use (NonCommercial, or CC-BY-NC) provided the original author and source are credited.

\section{INTRODUCTION}

The administration of antibiotics by injection may have some consequences as locoregional reactions such as skin necrosis at the injection site. Acute ischemia is very rare and occurs mainly when the injection is accidentally made intra-arterially. The first cases of accidental intra-arterial injection of drugs were reported in 1940 [1]. The drugs most associated with significant morbidity and/or mortality were barbiturates and benzodiazepines [2], and among antibiotics, flucloxacillin is the most implicated [3].

\section{Observation 1}

4-year-old child, without any notable pathological history, was hospitalized for extensive circumferential erysipelas of the right leg. A staphylococcal origin was evoked by the existence of a super-infected boil. The child was treated by flucloxacillin intravenous injection at a dose of $450 \mathrm{mg}$ every six hours, which was performed by the nurse without dilution, at the crease of the right elbow, accidentally intra-arterially. Four hours after the first injection, the child felt intense pain in the right hand with paleness, paralysis, coldness and leak of the radial pulse (Figure 1). No lesion was observed around the injection site. An echo-doppler exam of the right upper extremity vessels was carried out and showed that the ulnar and radial arteries were permeable. A curative heparin therapy, based on LMWH (Low Molecular Weight Heparin), was initiated. Enoxaparin was administered in 2 subcutaneous injections per day (each 12 hours), 100 IU Anti-Xa/kg each, for 20 days, in combination with aspirin at an anti-aggregant dose, and with elevation and massage of the limb. The evolution was favorable (disappearance of signs of ischemia at D3 of treatment) outside areas of superficial cutaneous necrosis which cicatrized later.

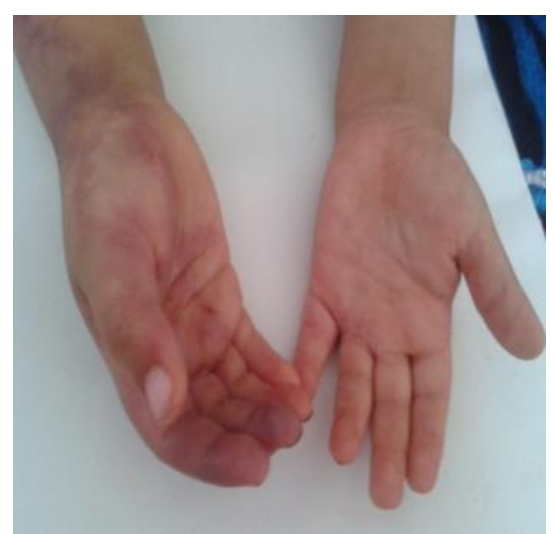

Fig-1: Appearance of the limb 6 hours after injection of floxacin

\section{OBSERVATION 2}

One-month old infant was admitted to neonatal resuscitation for respiratory distress due to cardiac malformation during hospitalization. The patient was 
put on penicillin-based antibiotics for pulmonary infection which were also accidentally injected directly into the arteries. 6 hours later he had developed cyanosis involving the entire forearm, with coldness, unperceived radial and ulnar pulses and skin necrosis opposite the injection site at the fold of the elbow and at the extremities (figure 2). The arterial Doppler ultrasound exam was performed, showing weak arterial flow in the radial and ulnar arteries without any proof of thrombus. The patient was placed on heparin sodium according to the following protocol: Attack dose of 50 to $100 \mathrm{U} / \mathrm{kg}$ followed by continuous infusion at an average dosage of $27 \mathrm{U} / \mathrm{kg} / \mathrm{h}$ over 40 minutes. The evolution has led to the disappearance of the signs of ischemia but the persistence of digital cutaneous necrosis around the injection site (figure 3 ). The patient underwent a necrosectomy one month later.

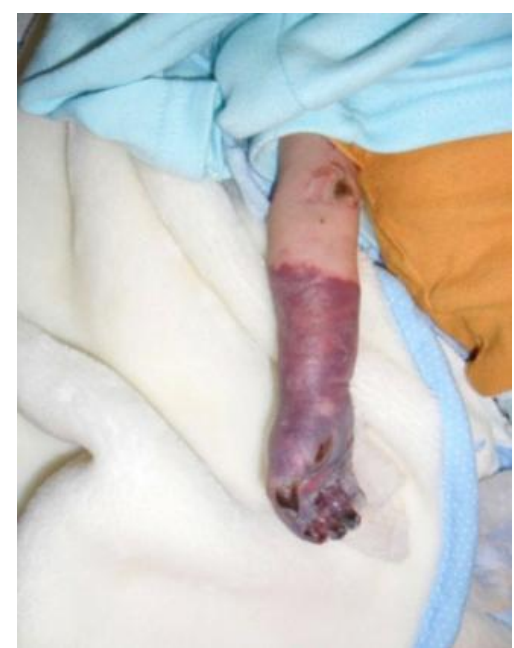

Fig-2: Acute ischemia after injection of antibiotics intra arterial

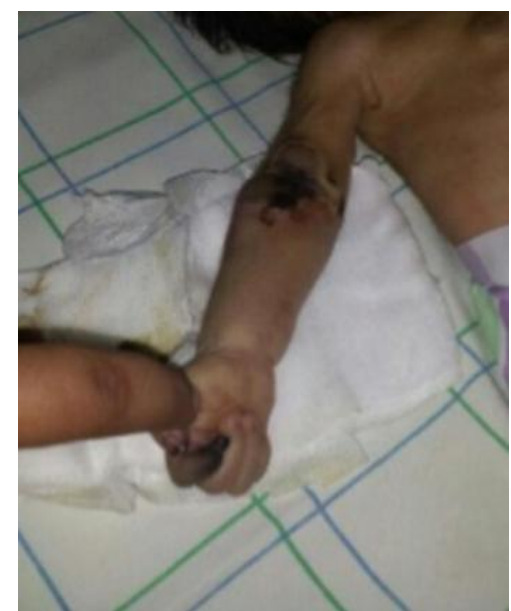

Fig-3: Clinical improvement after heparin therapy

\section{ObServation 3}

A 45-year-old patient without notable pathological history operated in the neurosurgery department for cerebral meningioma who had received postoperatively an antistaphilococcal drug, which was accidentally injected intra-arterially. The patient developed signs of ischemia with cyanosis digital necrosis, paralysis, and coldness and pulse suppression. The arterial Doppler ultrasound exam of the upper limb did not objectives any thrombus with permeable radial and ulnar arteries. Low molecular weight heparin was administered in 2 subcutaneous injections per day with each 12 hours, of 800 Anti-Xa IU/kg each, for 1 month, with persistence of digital necrosis, a necrosectomy was then performed 3 months later.

\section{DiscuSSION}

Accidental intra-arterial injection of antibiotics remains a rare event. Knowles reported injecting benzathine penicillin into a child's gluteal artery in 1966 [4]. The results were the same to the cases explained before, with immediate pain, mottling, cyanosis and skin necrosis that eventually recovered. Other cases have been reported where accidental intra-arterial injection of Antibiotics into the elbow resulted in endothelial damage and complete thrombosis of the entire distal arterial system.

Flucloxacillin and penicillin are the most implicated antibiotics. Several pathophysiological hypotheses have been put forward: flucloxacillin is responsible for the formation of micro-crystals in small vessels, intimal lesions may lead to hemolysis associated with platelet aggregation, venous obstruction may lead to stasis and thrombosis, and vasospasm may be reflex [5]. Furthermore, the size of penicillin crystals and the viscous aspect of the product led to the development of these reactions and flucloxacillin has been described as having phlebogenic properties, hence the importance of complying with the procedures for diluting the product in a sufficient volume and respecting good practices for intravenous administration of injectable products (aspiration before injection and slow injection).

Clinically, pain remains the main symptom, it is acute, followed by signs of ischemia, and should set a diagnosis.

There is no universally accepted therapeutic protocol. Some authors recommend limb elevation and massage, combined with heparin therapy [9]. Others suggest the use of lidocaine [10, 11] or hyperbaric oxygen therapy [12]. In some cases, regional nerve block or sympathectomy has been performed without great success [8, 9]. In another observation, surgical thrombectomy combined with heparin therapy was performed to limit the limb necrosis [6]. Finally, good results were obtained with intra-arterial injection of illoprost [10, 14], and in Bonnie's observation, he has good results using papaverine intra-arterially [9]. Unfortunately, the prognosis remains still blurring [9, 10].

Figure 4 deal with the different treatment options for intra-arterial injection of antibiotics. These cases were the subject of a national alert discussed at the level of the national pharmacovigilance 
commission, which amended the laboratory to add to the product's PCR, the thrombogenic risks and the need to comply with the product's dilution procedures. Dissemination of this information to the notification services made it possible to correct the product's dilution good practices.

In addition, cases of limb ischemia following the application of radial arterial lines in the resuscitation environment have been described in the literature, and the treatment was based on in situ thrombolysis, which also seems to give good results [14].

\section{Conclusion}

Although this complication is extremely rare, medical personnel have to be aware of the risk of gangrene after accidental intra-arterial injection of flucloxacillin. This complication can be avoided if the injection is performed into a vein, if the aspiration principle is respected, and finally if the injection is made slowly [2].

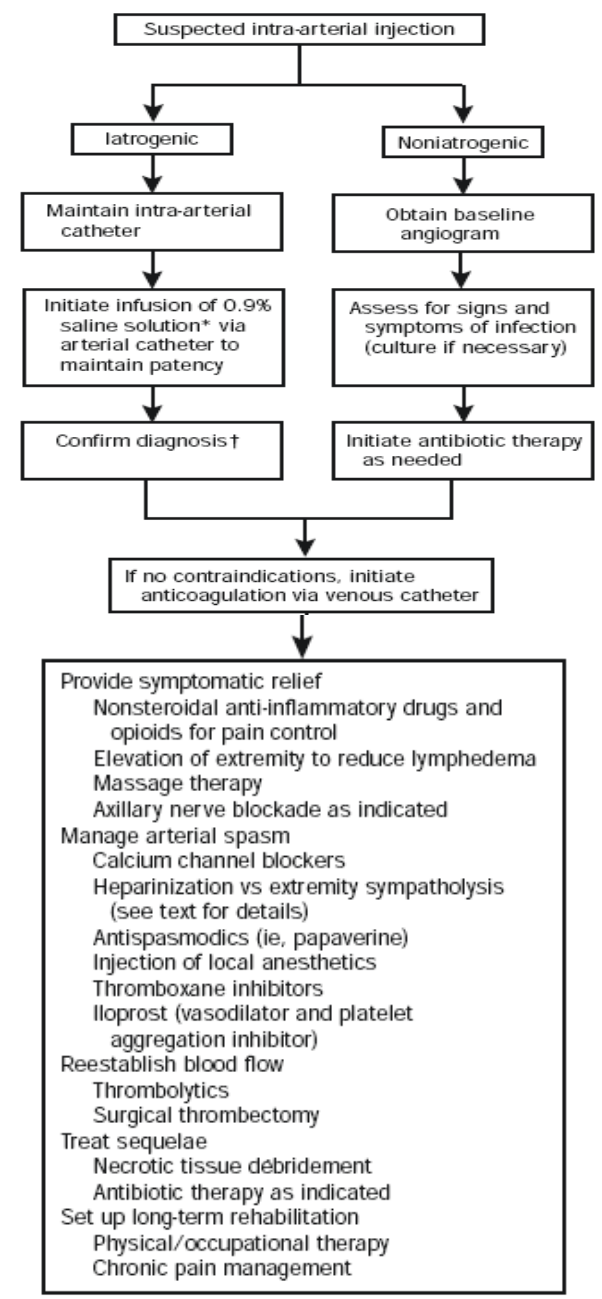

Fig-4: Algorithm of treatment of intra-arterial injections of antibiotics

\section{REFERENCE}

1. Macintosh RR, Heyworth PS. Intra-arterial injection of Pentothal: a warning. The Lancet. 1943 Nov 6;242(6271):571.

2. Cohen SM. Accidental intra-arterial injection of drugs. Lancet. 1948; 255:409-17.

3. Sen S, Nunes Chini E, Brown MJ. Complications after unintentional intraarterial injection of drugs: risks, outcomes, and management strategies. Mayo Clin Proc. 2005; 80:783-95.

4. Knowles JA. Accidental intra-arterial injection of penicillin. American Journal of Diseases of Children. 1966, 111 :552-6

5. Aghoutane EM, Fezzazi R, Elhaouati R, Boumzebra D. Gangrène des doigts après injection accidentelle en intra-artériel de floxacilline: à propos d'un cas. Chirurgie de la main. 2011 Apr 1;30(2):120-2.

6. Sen S, Nunes Chini E, Brown MJ. Complications after unintentional intraarterial injection of drugs: risks, outcomes, and management strategies. Mayo Clin Proc. 2005; 80:783-95

7. Benkirane R. Les modalités de dilution des produits injectables. Bulletin d'informations de pharmacovigilance Volume 5, Numéro $8 \& 9$, AoûtSeptembre. 2008

8. Arquilla B, Gupta R, Gernshiemer J, et al. Acute arterial spasm in an extremity caused by inadvertent intra-arterial injection successfully treated in the emergency department. J Emerg Med. 2000; 19:139-43.

9. Bhabra MS, Meshikhes AN, Thomson GJL. Intraarterial temazepam: an important cause of limb ischemia in intravenous drug abusers. Eur J Vasc Surg. 1994; $8: 240-2$.

10. Keene JR, Buckley KM, Small S. Accidental intraarterial injection: a case report, new treatment modalities, and a review of literature. $\mathrm{J}$ Oral Maxillofac Surg. 2006; 64 :965-8.

11. Ali MS, Chowdhary UM, Mohanna. Drug induced arterial spasm releaved by lidocaine. Acta Chir Scand 1986; 152 :697-9.

12. Adir Y, Halpern P, Nachum Z. Hyperbaric oxygen therapy for ischemia of the hand due to intraarterial injection of methadone and flunitrazepam. Eur J Vasc Surg. 1991; 5:677-9.

13. Tait IS, Holdsworth, Belch JJ. Management of intra-arterial injection injury with illoprost. Lancet. 1994; 343:419.

14. Jean-Franc, ois H. Geschwind MD, Mandeep S. Dagli MD, Drew L. Lambert MD and Hicham Kobeiter, MD, Department of Radiology, The Johns Hopkins Hospital, Baltimore, Maryland, USA, J Endovasc Ther. $2003 ; 10: 590-594$. 